\title{
Vibration Therapy as an Intervention for Enhancing Trochanteric Hip Fracture Healing in Elderly Patients: A Randomized Double-Blinded, Placebo- Controlled Clinical Trial
}

\section{Ronald Man Yeung WONG}

The Chinese University of Hong Kong

\section{Simon Kwoon Ho CHOW}

The Chinese University of Hong Kong

Ning TANG

The Chinese University of Hong Kong

Yik Lok CHUNG

The Chinese University of Hong Kong

James GRIFFITH

The Chinese University of Hong Kong

Wing Hong LIU

The Chinese University of Hong Kong

Raymond Wai Kit NG

The Chinese University of Hong Kong

\section{Chi Yin TSO}

The Chinese University of Hong Kong

Wing-Hoi Cheung ( $\square$ louischeung@cuhk.edu.hk)

The Chinese University of Hong Kong https:// orcid.org/0000-0003-3247-8255

Study protocol

Keywords: Hip fractures, Vibration treatment, Osteoporosis, Trochanteric fracture, Randomized controlled trial

Posted Date: June 28th, 2021

DOl: https://doi.org/10.21203/rs.3.rs-354202/v1

License: (c) (1) This work is licensed under a Creative Commons Attribution 4.0 International License. Read Full License 
Version of Record: A version of this preprint was published at Trials on December 1st, 2021. See the published version at https://doi.org/10.1186/s13063-021-05844-y. 


\section{Abstract}

\section{Background}

There are more than 300,000 hip fractures yearly in the US with mortality rates of $20 \%$ within 1 year. The treatment of osteoporotic fractures is a major challenge as bone quality is poor, and healing is expected to delay due to the impaired healing properties with respect to bone formation, angiogenesis and mineralization. Enhancement of osteoporotic fracture healing and function is therefore critical as a major goal in modern fracture management. Previous pre-clinical studies have shown that low-magnitude highfrequency vibration (LMHFV) accelerates osteoporotic fracture healing. The objective of this study is to investigate the effect of LMHFV on accelerating trochanteric hip fracture healing and functional recovery.

\section{Methods}

This is a randomized, double-blinded, placebo-controlled clinical trial to evaluate the effect of LMHFV in accelerating trochanteric hip fracture healing. All fractures undergo cephalomedullary nail fixation. The primary outcome of this study is time to fracture healing by X-ray. Computed tomography (CT) and Dualenergy X-ray absorptiometry (DXA) will also be performed. Blood circulation at the fracture site will be assessed by dynamic perfusion magnetic resonance (MR). Clinical results include functional recovery by muscle strength, timed up and go test (TUG), quality of life questionnaire (SF-36), balancing, falls, and mortality.

\section{Discussion}

Previous animal studies have demonstrated LMHFV to improve both normal and osteoporotic fracture healing by accelerating callus formation and mineralization. The mechanical stimulation stimulates angiogenesis by significantly enhancing vascular volume and blood flow velocity. This is the first study to translate LMHFV to enhancing hip fracture healing clinically. Positive results would provide a huge impact in the recovery of hip fracture patients and save healthcare costs.

\section{Trial registration}

Clinicaltrials.gov, NCT04063891. Registered on August 21, 2019

\section{Background}

Hip fractures are currently ranked as the top 10 causes of disability (1). The leading cause is due to osteoporosis and the lifetime fracture risk of osteoporotic patients reaches as high as $40 \%$ (2). There are approximately 2.5 million osteoporotic fractures each year in the United States (US), with costs estimated at 15 billion USD in 2010 (3). In fact, 300,000 hip fractures occur yearly in the US with mortality rates of $20 \%$ within 1 year (4). Furthermore, in the United Kingdom (UK), approximately 80,000 hip fractures are treated yearly with direct costs of 2 billion pounds, and mortality of $30 \%$ at 1 year (5). The World Health 
Organization (WHO) predicts that the aging population will continue to rise over the next 25 years, and therefore the socioeconomic burden is expected to grow rapidly.

The treatment of osteoporotic fractures is a major challenge as bone quality is poor, and healing is expected to delay due to the impaired healing properties with respect to bone formation, angiogenesis and mineralization (6-8). Failure to unite results in pain, weakness, reduced mobility and surgical fixation failure, and these complications are most common in elderly patients, which can lead to serious detrimental effects to overall health status. Furthermore, due to prolonged recovery, muscle function often deteriorates as well. Enhancement of osteoporotic fracture healing and function is therefore critical as a major goal in modern fracture management.

Low-magnitude high-frequency vibration (LMHFV) is a promising biophysical intervention that provides non-invasive, systemic mechanical stimulation. Previous animal studies have demonstrated LMHFV to improve both normal and osteoporotic fracture healing by accelerating callus formation and mineralization $(6,7,9,10)$. The mechanical stimulation stimulates angiogenesis by significantly enhancing vascular volume and blood flow velocity (7). Further investigating into the molecular pathways, we have shown LMHFV to enhance healing from the early inflammation stage to the late phases of remodeling $(7,10-13)$. Clinical trials have also shown positive improvements in reaction time, movement velocity, balancing ability and quadriceps muscle strength in healthy subjects (14), which were retained 1 year after cessation of LMHFV treatment (15).

With positive result from previous pre-clinical studies, we translate the use of LMHFV to enhancing hip fracture healing. The objective of this study was to assess the efficacy of LMHFV in accelerating trochanteric hip fracture healing.

\section{Methods}

\section{Study Design and Standardization of Surgical Management}

This is a randomized, double-blinded, placebo-controlled clinical trial to evaluate the effect of LMHFV (VH-001 exercise platform; V-health Limited, Hong Kong) on accelerating trochanteric hip fracture healing. Patients are recruited from the Prince of Wales Hospital, affiliated with the Chinese University of Hong Kong. Closed reduction and cephalomedullary nail fixation (Gamma3 nail, Stryker, USA) (19) will be performed for all patients within 48 hours of admission. Prophylactic intravenous antibiotic with Cefazolin $1 \mathrm{~g}$ is given before surgical incision and continued for 3 doses after. Postoperatively, pressure bandage will be given until mobilization. Adequate analgesics will be given. Full weight bearing is started as soon as condition allows.

\section{Inclusion Criteria}

The inclusion criteria are as follows:

1. Elderly male or females aged 65 years or older 
2. Unilateral trochanteric hip fractures (AO classification A1-A3)

3. Due to unintentional fall

4. Fractures fixed with cephalomedullary nail (Gamma nail, Stryker)

5. Willing and able to comply with study protocol

\section{Exclusion Criteria}

Exclusion criteria are as follows:

1. Open fracture

2. Bilateral fractures

3. Patient with multiple injuries

4. Pathological fractures e.g., tumour.

5. History of medication or disease affecting bone metabolism such as hypo/hyperthyroidism., etc.

6. Malignancy

7. Chairbound or bedbound

8. Cognitive problems e.g. dementia.

\section{Sample size}

In this study, time to radiologic healing is the primary objective. This is a well-accepted quantitative approach to evaluate fracture union, as in our previous study (16). We expect $20 \%$ acceleration of radiologic healing time (our animal data confirms $30 \%$ acceleration (9)). A sample size of 100 will detect a significant difference using ANOVA with $80 \%$ power and 0.05 significance level (PASS 11.0, NCSS, LLC, Utah, USA). Our previous clinical trial (14) confirmed that compliance of vibration treatment was very good with normal subjects at $66 \%$ on average (14). We conservatively increase the sample size to 120 , assuming $15 \%$ dropout rate.

\section{Randomization and blinding}

Patients will be recruited from the Department of Orthopaedics and Traumatology, Prince of Wales Hospital, Hospital Authority, Hong Kong, based on inclusion and exclusion criteria. According to our previously established protocol (17), a computer-generated set of random allocations are sealed in consecutively numbered opaque envelopes by an independent central technician. Once consent is obtained, the patient is randomized into either vibration or placebo group by opening the next sealed envelope. Patients and investigators are blinded to treatment assignment. Outcome assessors and statistician are blinded.

Patient demographics will be recorded and confirmed with the Clinical Management System (CMS), Hospital Authority, which is the central electronic database for public hospitals in Hong Kong (18). Before signing the consent form, each patient will be explained the objectives, benefits and risks of the study and 
their rights and responsibilities, as well as privacy and confidentiality information. An information sheet will also be distributed.

\section{Interventions}

Patients will be randomly allocated to placebo or vibration group (20). Vibration is treated with LMHFV (Vhealth Ltd, Hong Kong) at $35 \mathrm{~Hz}, 0.3 \mathrm{~g}$ (g = gravitational acceleration) for 20 minutes/day, 5 times/week (14) while placebo group will have sham treatment to stand on the LMHFV platform for 20 minutes/day, 5 times/week. Sound emitted by machine masks the noise an active machine makes (21). Treatment is started at 3 days post-operation for 6 months. Patients will be followed up to 12 months.

\section{Outcome assessments}

\section{Plain Radiographs}

Anteroposterior and lateral X-ray (DRX-Evolution, Carestream Health, Rochester, NY, USA) of fracture site will be performed at baseline, 6,12 , and 26 weeks post-operation. Films will be assessed by two blinded surgeons independently, to note healing in the AP or lateral film (16). Fracture healing is defined based on the Radiographic Union Score of Hip (RUSH), which assesses callus formation and visibility of fracture line at 4 cortices (22).

\section{Computed Tomography (CT)}

CT (SOMATOM Drive, Siemens, Erlangen, Germany) scan will be performed at the region of interest $5 \mathrm{~cm}$ proximal and distal to the fracture site (23), at baseline, 6 , and 12 weeks post-operation. Films will be assessed by a blinded radiologist.

\section{Clinical Monitoring}

Standard postoperative program for all in-patients, including vital sign monitoring, wound condition, neurovascular status, pain, analgesic and mobilization.

\section{Densitometry Status}

Bone Mineral Density (BMD) and Bone mineral content (BMC) will be measured at baseline, 6, 12 and 26 weeks post-operation using DXA (Horizon DXA System, Hologic Inc, USA) to monitor changes in bone quality at the fracture site (15).

\section{Dynamic Perfusion MRI}

Blood circulation at fracture site will be measured at baseline and 6 weeks post-operation with dynamic contrast-enhanced MR imaging (Intera NT; Philips Medical Systems, Best, Netherlands) with maximum gradient strength of $30 \mathrm{mT} / \mathrm{m}$ as in our previously established protocol $(24,25)$. 


\section{Functional and Quality of Life Outcomes}

At $6,12,16,26,52$ weeks post-operation, all patients will be assessed with the following functional tests:

\section{Verbal Descriptor Scale}

Verbal descriptor scale will be used to assess different levels of pain intensity, which has good reliability and validity in elderly patients (26).

\section{Quality of Life Short Form-36 (SF-36)}

SF-36 is a well-validated functional status questionnaire to measure health-related quality of life in eight domains, including physical functioning, health perception, etc. A higher scores indicates better function (14).

\section{Quadriceps Muscle Strength}

Quadriceps muscle strength will be measured on the affected limb with an isometric dynamometer (Baseline, Genova, Italy). Subject will sit on a chair with both feet above ground, while raising the affected leg $45^{\circ}$ forwards. The dynamometer will be placed above the ankle and the subject will push the leg forward with maximum force $(\mathrm{N})$. Measurements will be repeated three times and the maximum value will be used for evaluation (14).

\section{Balancing Ability}

To assess balancing ability, the Biodex Balance System SD (Biodex Medical Systems Inc., Shirley, NY, USA) is used to measure the static and dynamic ability of patients to maintain centre of balance. The score generated by the machine assesses the deviation from centre via an Overall Stability Index (OSI), Anterior/Posterior Stability Index (APSI) and Medial/Lateral Stability Index (MLSI), which have been shown to be reliable tools for objective assessment of postural stability in several studies in elderly patients (20).

\section{Times Up and Go (TUG) test}

The TUG test will be used to test basic mobility skills, which is a useful predictor of risk of falls. The patient will stand from a chair, walk 3 meters and travel back and sit back on the chair. The time in seconds (s) is recorded (20).

\section{Falls}

Mortality will be measured at 1 month, 3 months and 1 year via a fall calendar, which is well established in clinical trials for elderly patients (14).

\section{Mortality}


Mortality will be measured at 1 month, 3 months and 1 year (27). Mortality is documented from the Clinical Management System (CMS) from Hospital Authority, a central public computerized system for patients in Hong Kong (18).

\section{Compliance assessment}

A smart card is also given to each participant to record compliance to placebo and LMHFV (14).

\section{Adverse Event Handling}

Currently, there is no report of adverse event in the use of LMHFV. In case of unusual conditions including intolerable pain, LMHFV treatment will be terminated and clinicians will follow-up the patient. Any adverse events or problems during the study are recorded by an independent staff member.

\section{Data Collection and Management}

The research assistant will be trained to ensure accuracy of outcome assessments and data collection. The ethics committee will oversee any issues raised and corresponding measures will be taken if necessary. Subjects not willing to continue can withdraw at any time. The study will comply with the good clinical practice guidelines. Each patient will be assigned an identification code. The patient identification code list and database will be safeguarded.

\section{Statistics}

All results will be expressed in mean \pm standard deviation. Statistical analysis will be performed using SPSS (IBM, NY, USA). One-way (for between-group differences in over-time changes), two-way (for interaction effect among time and group) and group repeated measures ANOVA with post-hoc Bonferroni analysis, as well as independent/paired t-tests will be performed. Significance level is set at $p<0.05$ (2tailed).

\section{Dissemination Plans}

We will disseminate the trial results to healthcare professionals, the public, and other relevant groups as soon as results are available.

\section{Discussion}

This is the first double-blinded, placebo-controlled trial to investigate the efficacy of LMHFV in accelerating trochanteric hip fracture healing. With the current aging population and increasing number of hip fractures, strategies to enhance healing and functional recovery is crucial. LMHFV is a non-invasive treatment, which is easily acceptable for elderlies and has proven benefits for physical function in a previously conducted randomized controlled trial of 710 healthy, active and independent postmenopausal women (14). Benefits of LMHFV for balancing ability, muscle strength and risk of falling were retained 1 year after cessation of the treatment (15). No serious side effects were noted. Numerous animal studies 
have also shown LMHFV to significantly improve normal and osteoporotic fracture healing with increased callus formation, angiogenesis and mineralization $(6,7,9,10)$. However, clinical translation in hip fractures remains to be proven.

Current Fracture Liaison Services (FLS) aims to close the osteoporosis care gap and prevent secondary fractures that can lead to high morbidity and mortality (28). However, the presence of sarcopenia often co-exists with osteoporosis leading to the geriatric syndrome "osteosarcopenia" (29). The prevalence of sarcopenia also reaches up to $95 \%$ in males and $64 \%$ in females in osteoporotic fracture patients (30). It has been postulated that most patients develop muscle deterioration during immobilization from a fracture. Therefore, the incorporation of effective strategies to accelerate fracture healing for early painfree mobilization has been advocated.

The risk of an imminent fracture is also high after an initial osteoporotic fracture. In fact, more than $50 \%$ of secondary fractures occur during the first 2 years (31). Therefore, this window of opportunity is also a golden period for intervention. Positive results of LMHFV in enhancing fracture healing and functional outcome would have a significant impact for hip fracture patients by decreasing disability. More importantly, the incorporation of LMHFV into FLS can potentially lead to a significant decrease in health care costs.

Enrolment in this trial is planned to begin in January 2021. Completion is expected to take 24 months. We speculate that positive results would allow the incorporation of LMHFV into hip fracture multidisciplinary rehabilitation programs.

The completion of the trial is expected to take 24 months.

\section{Declarations}

\section{Trial status}

The trial was initially planned to start on January 2020. However, due to the COVID-19 situation, the trial has not yet started. Enrolment in this trial is planned to begin in January 2021.

\section{Ethics approval and consent to participate}

This study has been approved by The Joint Chinese University of Hong Kong - New Territories East Cluster Clinical Research Ethics Committee (CREC Ref No: 2018.584). All study related documents and computerized processed data will be handled as strictly confidential under the Good Clinical Practice Guidelines and all data collected from this study will be used for the purpose of this study only.

Patient identity will not be revealed in any publication or report generated from this study. Direct access to participants' records will be given to investigators and authorized research staff only. Direct access will also be given to members of Regulatory Authorities and Ethics Committees. 
Informed consent will be obtained from all study participants.

- Consent for publication: Not applicable

- Availability of data and materials: Please contact author for data requests.

- Competing interests: The authors declare that they have no competing interests.

- Funding: The study was supported by the General Research Fund Early Career Scheme, HKSAR Research Grant Council (Ref: 24108519).

- Author Contributions: RMYM, WHC, SKHC contributed to study design; RMYM, NT, YLC, JG, WHL, RWKN, CYT contributed to data collection; RMYM, WHC, SKHC contributed to data analysis, while RMYM, WHC, SKHC data interpretation; RMYW, WHC contributed to manuscript writing.

\section{References}

1. Bhandari M, Swiontkowski M. Management of Acute Hip Fracture. N Engl J Med. 2017;377(21):2053-62.

2. Rachner TD, Khosla S, Hofbauer LC. Osteoporosis: now and the future. Lancet. 2011;377(9773):1276-87.

3. Solomon DH, Patrick AR, Schousboe J, Losina E. The potential economic benefits of improved postfracture care: a cost-effectiveness analysis of a fracture liaison service in the US health-care system. Journal of bone mineral research: the official journal of the American Society for Bone Mineral Research. 2014;29(7):1667-74.

4. Black DM, Rosen CJ. Postmenopausal Osteoporosis. N Engl J Med. 2016;374(21):2096-7.

5. Baker PN, Salar O, Ollivere BJ, Forward DP, Weerasuriya N, Moppett IK, et al. Evolution of the hip fracture population: time to consider the future? A retrospective observational analysis. BMJ Open. 2014;4(4):e004405.

6. Shi HF, Cheung WH, Qin L, Leung AH, Leung KS. Low-magnitude high-frequency vibration treatment augments fracture healing in ovariectomy-induced osteoporotic bone. Bone. 2010;46(5):1299-305.

7. Cheung WH, Sun MH, Zheng YP, Chu WC, Leung AH, Qin L, et al. Stimulated angiogenesis for fracture healing augmented by low-magnitude, high-frequency vibration in a rat model-evaluation of pulsedwave doppler, 3-D power Doppler ultrasonography and micro-CT microangiography. Ultrasound Med Biol. 2012;38(12):2120-9.

8. Wong RMY, Choy MHV, Li MCM, Leung KS, K-H Chow S, Cheung WH, et al. A systematic review of current osteoporotic metaphyseal fracture animal models. Bone Joint Res. 2018;7(1):6-11.

9. Leung KS, Shi HF, Cheung WH, Qin L, Ng WK, Tam KF, et al. Low-magnitude high-frequency vibration accelerates callus formation, mineralization, and fracture healing in rats. $J$ Orthop Res. 2009;27(4):458-65. 
10. Chung SL, Leung KS, Cheung WH. Low-magnitude high-frequency vibration enhances gene expression related to callus formation, mineralization and remodeling during osteoporotic fracture healing in rats. J Orthop Res. 2014;32(12):1572-9.

11. Chow DH, Leung KS, Qin L, Leung AH, Cheung WH. Low-magnitude high-frequency vibration (LMHFV) enhances bone remodeling in osteoporotic rat femoral fracture healing. J Orthop Res. 2011;29(5):746-52.

12. Chow SK, Chim YN, Wang J, Zhang N, Wong RMY, Tang N, Leung KS, Cheung WH. Vibration Treatment Modulates Macrophage Polarization and Enhances Early Inflammatory Response in Estrogen-deficient Osteoporotic Fracture Healing (Submitted). J Orthop Res. 2018.

13. Wei FY, Chow SK, Leung KS, Qin J, Guo A, Yu OL, et al. Low-magnitude high-frequency vibration enhanced mesenchymal stem cell recruitment in osteoporotic fracture healing through the SDF1/CXCR4 pathway. Eur Cell Mater. 2016;31:341-54.

14. Leung KS, Li CY, Tse YK, Choy TK, Leung PC, Hung VW, et al. Effects of 18-month low-magnitude high-frequency vibration on fall rate and fracture risks in 710 community elderly-a clusterrandomized controlled trial. Osteoporos Int. 2014;25(6):1785-95.

15. Cheung WH, Li CY, Zhu TY, Leung KS. Improvement in muscle performance after one-year cessation of low-magnitude high-frequency vibration in community elderly. J Musculoskelet Neuronal Interact. 2016;16(1):4-11.

16. Leung KS, Lee WS, Tsui HF, Liu PP, Cheung WH. Complex tibial fracture outcomes following treatment with low-intensity pulsed ultrasound. Ultrasound Med Biol. 2004;30(3):389-95.

17. Wong RM, Tang N, Tso CY, Ho WT, Ng WK, Chow SK, et al. Vibration Therapy as an Intervention for Postural Training and Fall Prevention after Distal Radius Fracture in Elderly Patients: A Randomized Controlled Trial (Submitted). The Hong Kong Orthopaedic Association The 38th Annual Congress; Hong Kong2018.

18. Cheung NT, Fung KW, Wong KC, Cheung A, Cheung J, Ho W, et al. Medical informatics-the state of the art in the Hospital Authority. Int J Med Inform. 2001;62(2-3):113-9.

19. Leung KS, So WS, Shen WY, Hui PW. Gamma nails and dynamic hip screws for peritrochanteric fractures. A randomised prospective study in elderly patients. J Bone Joint Surg Br. 1992;74(3):34551.

20. Wong RMY, Ho WT, Tang N, Tso CY, Ng WKR, Chow SK, et al. A study protocol for a randomized controlled trial evaluating vibration therapy as an intervention for postural training and fall prevention after distal radius fracture in elderly patients. Trials. 2020;21(1):95.

21. Kiel DP, Hannan MT, Barton BA, Bouxsein ML, Sisson E, Lang T, et al. Low-Magnitude Mechanical Stimulation to Improve Bone Density in Persons of Advanced Age: A Randomized, PlaceboControlled Trial. J Bone Miner Res. 2015;30(7):1319-28.

22. Morshed S. Current Options for Determining Fracture Union. Adv Med. 2014;2014:708574.

23. Griffith JF, Genant HK. New imaging modalities in bone. Curr Rheumatol Rep. 2011;13(3):241-50. 
24. Griffith JF. Functional imaging of the musculoskeletal system. Quant Imaging Med Surg. 2015;5(3):323-31.

25. Griffith JF, Yeung DK, Antonio GE, Lee FK, Hong AW, Wong SY, et al. Vertebral bone mineral density, marrow perfusion, and fat content in healthy men and men with osteoporosis: dynamic contrastenhanced MR imaging and MR spectroscopy. Radiology. 2005;236(3):945-51.

26. Herr KA, Garand L. Assessment and measurement of pain in older adults. Clin Geriatr Med. 2001;17(3):457-78. vi.

27. Lisk R, Yeong K. Reducing mortality from hip fractures: a systematic quality improvement programme. BMJ Qual Improv Rep. 2014;3(1).

28. Wong RMY, Law SW, Lee KB, Chow SKH, Cheung WH. Secondary prevention of fragility fractures: instrumental role of a fracture liaison service to tackle the risk of imminent fracture. Hong Kong Med J. 2019;25(3):235-42.

29. Hirschfeld HP, Kinsella R, Duque G. Osteosarcopenia: where bone, muscle, and fat collide. Osteoporos Int. 2017;28(10):2781-90.

30. Wong RMY, Wong H, Zhang N, Chow SKH, Chau WW, Wang J, et al. The relationship between sarcopenia and fragility fracture-a systematic review. Osteoporos Int. 2019;30(3):541-53.

31. Johansson H, Siggeirsdóttir K, Harvey NC, Odén A, Gudnason V, McCloskey E, et al. Imminent risk of fracture after fracture. Osteoporos Int. 2017;28(3):775-80.

\section{Figures}




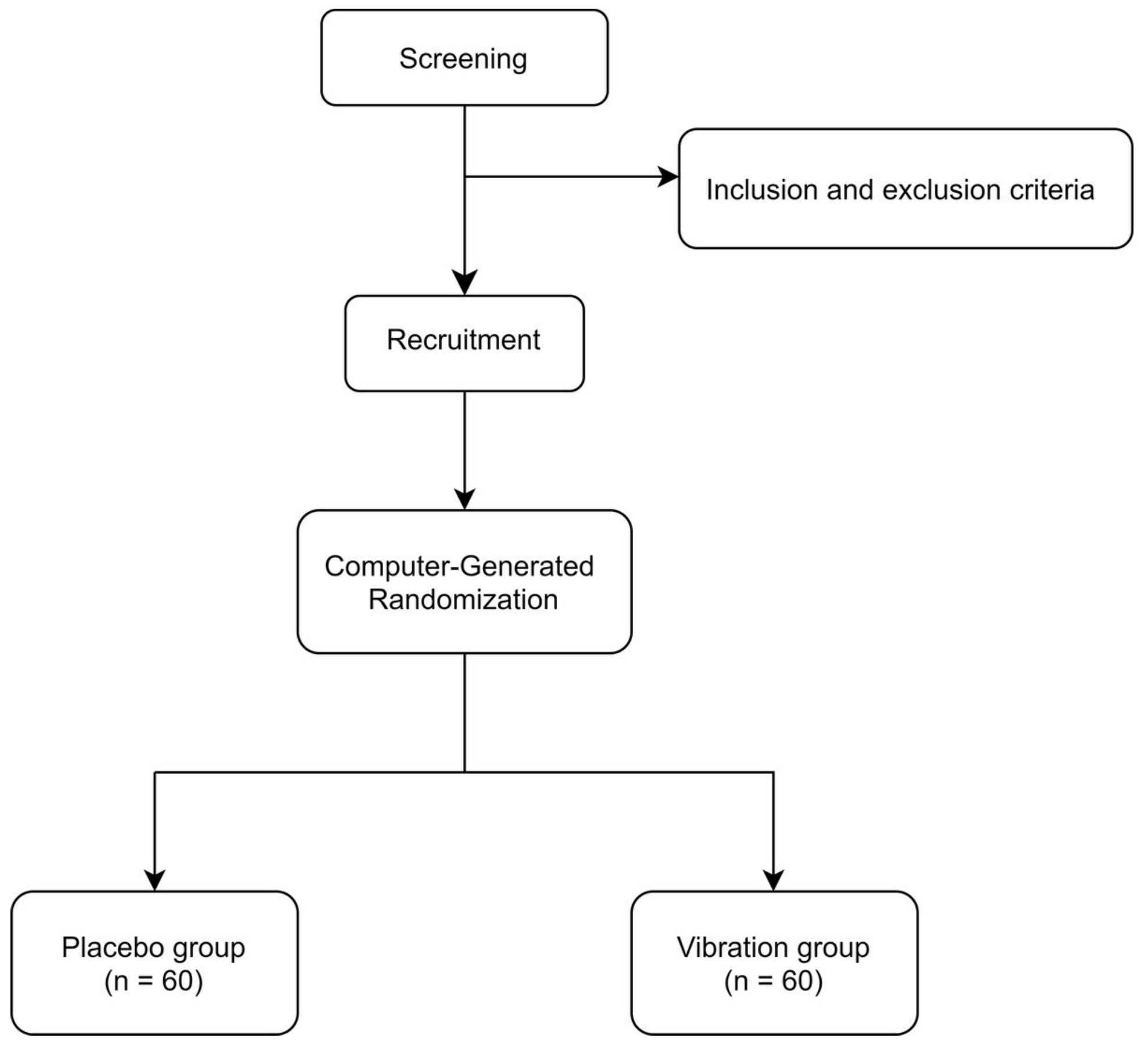

Figure 1

shows a flowchart of the study design. The Consolidated Standards of Reporting Trials (CONSORT) checklist is provided as Additional file 1; the Standard Protocol Items: Recommendations for Interventional Trials (SPIRIT) checklist is presented in Additional file 2.

\section{Supplementary Files}

This is a list of supplementary files associated with this preprint. Click to download.

- CONSORTChecklistv1.doc 
- SpiritChecklistv2.doc

Page 14/14 